\section{Prostatakrebs junger Männer ist besonders gefährlich}

\author{
Prostatakrebs, der vor dem 50. Geburtstag diagnostiziert wird, gilt als beson- \\ ders aggressiv. Eine Studie hat gezeigt: Das stimmt - aber nur zum Teil.
}

$\mathrm{N}$ ur rund $1 \%$ der Männer, die an einem Prostatakarzinom erkranken sind jünger als 50 Jahre. Schon vor mehr als 30 Jahren legte eine Studie nahe, dass deren Prognose besonders schlecht ist. In der Folgezeit mehrten sich die Hinweise. Sie bezogen sich jedoch auf Männer, deren Erkrankung vor der Einführung des PSA-Tests diagnostiziert worden war.

$\mathrm{Ob}$ sich die damaligen Befunde auch in der PSA-Ära bestätigen, hat ein Team um Andreas Thorstenson vom Karolinska-Institut in Stockholm untersucht. Sie stellten dazu 919 Männer, deren Prostatakarzinom im Alter zwischen 35 und 49 Jahren erkannt worden war, rund 45.000 Männern gegenüber, deren Krebsdiagnose zwischen 50 und 66 Jahren gestellt worden war. Verglichen wurden die prostatakrebsspezifischen Mortalitätsraten.
$60 \%$ der Diagnosen vor dem Alter von 50 betrafen das Stadium T1c, gingen also kaum auf Symptome als vielmehr auf PSA-Testergebnisse zurück. Das Verhältnis von nicht metastasierten zu metastasierten Stadien war in allen Altersgruppen gleich. Die Diagnose eines metastasierten Prostatakarzinoms vor einem Alter von 50 Jahren war tatsächlich mit einer signifikant schlechteren Prognose assoziiert. Im Vergleich zur Sterblichkeit von Männern zwischen 50 und 59 Jahren war die krebsspezifische Sterblichkeit im unmittelbaren Vergleich um $41 \%$, nach Abgleich bestimmter Tumormerkmale um $28 \%$ erhöht. Für nicht metastasierte Karzinome nahm die Sterblichkeit mit dem Alter zu. Nach entsprechendem Abgleich war die krebsspezifische Mortalität hingegen in allen Altersgruppen ähnlich.

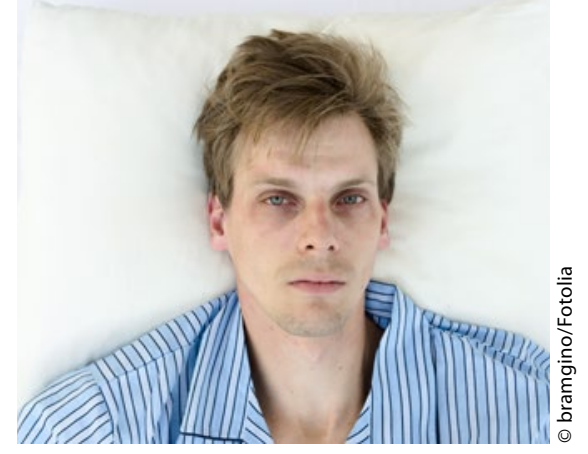

Vor allem bei Patienten unter 50 Jahren sind metastasierte Prostatakarzinome äußerst aggressiv.

Fazit: Thorstenson und Kollegen sehen ihre Befunde als Beleg dafür, dass eine aggressive Form des metastasierten Prostatakarzinoms in der Altersgruppe unter 50 besonders verbreitet ist. Sie fordern genetische Studien und Untersuchungen, die sich mit einer intensivierten systemischen Therapie in dieser Gruppe von Patienten beschäftigen.

Dr. Robert Bublak

Thorstenson A et al. Cancer-specific mortality in men diagnosed with prostate cancer before age 50 years, a nationwide population-based study. J Urol. 2016;doi: 10.1016/j.juro.2016. 06.080

\section{Je schwächer die Nieren, desto weniger nützen Statine}

\begin{abstract}
Ob bei ausgeprägter Niereninsuffizienz eine Therapie mit Statinen von Nutzen ist, hat die Cholesterol Treatment Trialists' (CTT) Collaboration untersucht.
\end{abstract}

W issenschaftler der CTT haben in einer Metaanalyse von 28 Studien den Einfluss der Statintherapie auf die Rate kardiovaskulärer Zwischenfälle mit Blick auf eine eingeschränkte Nierenfunktion untersucht. Beteiligt waren mehr als 180.000 Patienten mit unterschiedlichen Graden von Niereninsuffizienz. Insgesamt verringerte die statinbasierte lipidsenkende Therapie signifikant das Risiko für eine erste vaskuläre Komplikation, definitionsgemäß für einen tödlichen oder nicht tödlichen Herzinfarkt, die Notwendigkeit eines revaskularisierenden Eingriffs an den Koronarien, einen Schlaganfall und die vaskulär oder nicht vaskulär bedingte Sterblichkeit. Allerdings reduzierte sich der risikomin- dernde Effekt, relativ betrachtet, mit sinkender glomerulärer Filtrationsrate $(\mathrm{p}=$ 0,008 für den Trend). Lag die eGFR $\geq 60 \mathrm{ml} / \mathrm{min}$, ging das Risiko für vaskuläre Erstereignisse um 22 \% zurück. Bei einer eGFR zwischen 45 und $60 \mathrm{ml} / \mathrm{min}$ betrug die Reduktion $24 \%$. Danach nutzte sich der Schutzeffekt ab, er lag bei $-15 \%$ für eine eGFR $<45 \mathrm{ml} / \mathrm{min}$ und $<30 \mathrm{ml} /$ min ohne Dialysepflicht. Benötigten die Patienten eine Dialyse, war keine signifikante Risikosenkung mehr vorhanden.

In einem Kommentar zur Metaanalyse geben Muh Geot Wong und Vlado Perkovic, Universität Sydney, Australien, zu bedenken, dass sich zwar die relative Risikosenkung mit fallender eGFR abschwächt. Allerdings bedeute dies bei gleichzeitig steigender Häufigkeit vaskulärer Komplikationen, dass die absolute Reduktion der Gefahr potenziell etwa gleich bleibe. Die Zahl der zu behandelnden Patienten, um ein Ereignis zu verhindern, erhöhe sich damit nicht.

Fazit: Ob der relativ geringe Nutzen einer Statintherapie bei Nierenkrankheiten im Endstadium tatsächlich auf ein mangelndes Ansprechen zurückgehe, oder ob der Effekt in einer steigenden Zahl nicht durch Statine beeinflussbarer Ereignisse wie Arrhythmien und Herzversagen untergehe, sei unklar. Zudem verweisen Wong und Perkovic auf Studien, die einen positiven Einfluss von Statinen auf die Nierenfunktion von Patienten mit chronischen Nierenerkrankungen gezeigt hätten. Dr. Robert Bublak

Cholesterol Treatment Trialists' (CTT) Collaboration. Impact of renal function on the effects of LDL cholesterol lowering with statin-based regimens: a meta-analysis of individual participant data from 28 randomised trials. Lancet Diabetes Endocrinol. 2016; pii: S22138587(16)30156-5. 